\title{
L'EUROPE AU TABLEAU NOIR.
}

Comment les instituteurs français enseignent-ils l'Union européenne aujourd'hui ? Géraldine Bozec

\section{L'Harmattan | « Politique européenne »}

2010/1 n³0 | pages 153 à 186

ISSN 1623-6297

ISBN 9782296120372

Article disponible en ligne à l'adresse :

http://www.cairn.info/revue-politique-europeenne-2010-1-page-153.htm

\section{Pour citer cet article :}

Géraldine Bozec, « L'Europe au tableau noir. Comment les instituteurs français enseignent-ils l'Union européenne aujourd'hui ? », Politique européenne 2010/1 (n 30), p. 153-186.

DOI 10.3917/poeu.030.0153

Distribution électronique Cairn.info pour L'Harmattan.

(c) L'Harmattan. Tous droits réservés pour tous pays.

La reproduction ou représentation de cet article, notamment par photocopie, n'est autorisée que dans les limites des conditions générales d'utilisation du site ou, le cas échéant, des conditions générales de la licence souscrite par votre établissement. Toute autre reproduction ou représentation, en tout ou partie, sous quelque forme et de quelque manière que ce soit, est interdite sauf accord préalable et écrit de l'éditeur, en dehors des cas prévus par la législation en vigueur en France. Il est précisé que son stockage dans une base de données est également interdit. 
Géraldine BozEC

\section{L'EUROPE AU TABLEAU NOIR. \\ COMMENT LES INSTITUTEURS FRANÇAIS ENSEIGNENT-ILS L'UNION EUROPÉENNE AUJOURD'HUI ?'}

Cet article propose une analyse de la manière dont l'Union européenne est enseignée aujourd'hui par les instituteurs français. Il montre que les programmes scolaires ne mettent pas en reliefl'Union européenne comme communauté politique, la présentant essentiellement comme une collection de pays sans signification spécifique. Ils ancrent de manière prévalente le cadre national dans les repères des enfants, tout en faisant une nouvelle place à un universalisme associé désormais au monde entier. Les instituteurs témoignent du même tropisme national dans leur enseignement, mais cette attitude relève avant tout de routines professionnelles car des clivages idéologiques importants existent entre eux sur la nation et sur son importance. Des différences d'attitudes envers l'Union européenne sont aussi repérables, mais elles se heurtent au poids des programmes scolaires et des ouvrages pédagogiques, au refus de faire entrer la politique dans la classe et au caractère relativement flou du projet européen aux yeux des instituteurs. Au final, ces logiques combinées rendent l'UE difficile à traduire sur un plan pédagogique autrement quien la présentant à travers les pays qui la composent.

\section{Europe on the blackboard.}

\section{How do French primary teachers teach the European Union today?}

This article offers an analysis of the way French primary teachers teach Europe today. It shows that the school curriculum does not emphasize the EU as a political community, but rather presents it as a collection of countries without a specific signification. The curriculum emphasizes the prevalence of the national frame in the

\footnotetext{
${ }^{1}$ Je tiens tout particulièrement à remercier Sophie Duchesne, pour ses lectures attentives et ses commentaires sur ce texte. Je remercie également Sylvie Strudel, pour ses remarques sur la toute première version de ce texte, présenté au deuxième colloque international de la Section d'études européennes de l'Association française de science politique, intitulé "Amours et désamours entre Européens. Pour une sociologie politique des sentiments dans l'intégration européenne " (Grenoble, 6-7 décembre 2007).
}

politique européenne, $\mathrm{n}^{\circ} 30,2010$, p. 153-186. 
orientations of children, whilst according a new place to a vision of universalism now related to the global world. The teachers themselves demonstrate this same orientation towards the national level in their teaching, but this attitude is more a result of professional routines because significant ideological cleavages exist between them concerning the nation and its importance. It is also possible to identify different attitudes towards the EU, but these attitudes come into conflict with the weight of the school curricula and teaching materials. They also conflict with the essentially blurry notion of the European project in their eyes and the refusal to engage in politics in the classroom. Finally, the combination of these different rationales renders the EU difficult to transpose into a pedagogic project beyond simply presenting it via its different constituent countries.

\section{Introduction}

Le système éducatif a joué un rôle décisif, en France comme ailleurs, dans la formation et le renforcement de l'État nation (Green, 1992). Servant l'essor du capitalisme (Gellner, 1989), le développement d'une culture nationale par l'école visait également un objectif politique: définir l'espace et les formes du pouvoir et de l'identité politiques en encourageant une allégeance nationale et en familiarisant les élèves avec leur futur rôle de citoyens (Déloye, 1994, 2007; Thiesse, 2001, 240242). Cette mission de l'école a pris un relief particulier en France. Conçue comme un microcosme de l'espace public légitime, l'institution scolaire a été un des lieux centraux où se sont cristallisés les conflits sur le régime politique, et au-delà, sur la définition de la citoyenneté et de la nation (Déloye, 1994).

Réaffirmée avec force un siècle plus tard, à partir des années 1980, la mission civique de l'école française s'inscrit aujourd'hui dans un contexte très différent. La construction européenne, mais aussi la mondialisation et l'essor des discours et des mobilisations relatifs aux droits de l'homme tendent à élargir l'espace de l'identité et de l'action civiques des citoyens. L'orientation nationale de la socialisation civique scolaire se trouve ainsi questionnée (Schissler et Soysal, 2005).

Les interrogations sur la contribution de l'école au développement de la citoyenneté européenne se sont multipliées, au niveau communautaire et national, particulièrement depuis les années 1990 qui ont vu la consécration officielle d'une " citoyenneté de l'Union ». Les institutions communautaires se sont saisies du sujet de l'éducation à la citoyenneté 
européenne avec beaucoup de prudence, tant les réticences des États membres à l'égard d'un domaine - l'éducation - touchant à la construction de leur identité culturelle et politique étaient fortes. Pour autant, elles ont tenté de soutenir des actions éducatives, à partir de la fin des années 1980, permettant de renforcer la "dimension européenne dans l'éducation" et notamment la sensibilisation des enseignants et des élèves à l'identité et à la citoyenneté européennes². (Baeyens, 2000; Girod, 2006).

Comment les autorités éducatives et les enseignants français se sontils adaptés de leur côté à cette nouvelle réalité constituée par la construction européenne et le développement d'une citoyenneté européenne? La présente analyse ${ }^{3}$ porte sur la manière dont l'Union européenne est enseignée dans les dernières classes du primaire ${ }^{4}$, en s'intéressant en premier lieu aux disciplines scolaires qui ont été au cœur de l'élaboration du message civique délivré par l'école: l'histoire, la géographie et l'éducation civique.

Le contenu civique de l'enseignement élémentaire est plus rarement étudié que celui de l'enseignement secondaire. Pourtant, sans préjuger du maintien à l'identique des représentations et des attitudes qui se forment pendant l'enfance, on peut faire l'hypothèse, en raison même de leur antériorité, qu'elles ont un poids non négligeable sur la socialisation politique future (Percheron, 1978, 11-46). Peut-on donc relever, chez les acteurs scolaires, une volonté de promouvoir l'Europe dès l'école élémentaire comme nouvel espace d'identité et de citoyenneté

\footnotetext{
2 La résolution adoptée par le Conseil des Ministres de l'éducation de la Communauté européenne en 1988 (Journal officiel, $\mathrm{n}^{\circ} \mathrm{C} 177$ du 06/07/1988, p. 0005-0007) est la première à reconnaître officiellement la nécessité de " renforcer chez les jeunes le sens de l'identité européenne » et à énoncer des mesures en la matière.

3 Elle s'appuie sur les résultats d'une recherche doctorale en cours qui porte plus globalement sur le type de communauté politique qui est valorisée à l'école élémentaire, à travers les discours des acteurs du champ éducatif et les instructions officielles d'une part, les perceptions et les pratiques des instituteurs d'autre part. Pour plus de détails, voir la thèse en cours d'achèvement (titre provisoire: La République sur ses gardes. L'apprentissage de la citoyenneté à l'école élémentaire dans les discours et les pratiques contemporains, thèse pour l'obtention du doctorat de science politique, Institut d'études politiques de Paris). ${ }^{4}$ Ce sont les programmes de 2002 qui sont ici au cœur de l'analyse. Ils étaient en effet en vigueur au moment où l'enquête de terrain auprès des enseignants a été réalisée. Le champ d'étude est restreint au cycle 3 (CE2, CM1, CM2); l'histoire et la géographie n'existent pas en tant que telles, comme véritables disciplines, avant le CE2.
} 
communes? Hier hussards de la République, les instituteurs sont-ils devenus aujourd'hui des militants de l'Union européenne?

Avant d'examiner leurs pratiques d'enseignement et d'essayer d'en rendre compte, on présentera un des éléments centraux susceptibles d'informer ces pratiques: ce qui leur est prescrit en la matière par les instructions officielles. Comment les programmes scolaires traitent-ils de l'enseignement de l'Europe?

\section{L'Europe dans les programmes scolaires}

\section{Une collection d'États}

Les contenus de l'enseignement primaire ont peu évolué de la Troisième République aux premières décennies qui ont suivi la Deuxième Guerre mondiale. L'une des finalités majeures de cet enseignement était de diffuser une connaissance globale de la nation et de promouvoir l'attachement envers celle-ci (Ozouf, 1964; Fumat, 1978). L'histoire enseignée véhiculait l'idée d'une France immémoriale, enracinée dans une origine gauloise, élargissant progressivement son espace grâce aux rois bâtisseurs de territoire et d'État, et devenant à partir de la Révolution l'incarnation, privilégiée entre les nations, des principes démocratiques et des droits de l'homme (Citron, 2008; Ferro, 1981; Nora, 1986; Perrot, 1973). L'éducation civique visait quant à elle à inculquer certaines vertus de la morale individuelle et à encourager l'attachement à la patrie et les devoirs envers celle-ci (Déloye, 1994; Baubérot, 1997). De son côté, la géographie scolaire apprenait aux enfants à reconnaître un espace national bien délimité, varié d'un " petit pays » à l'autre, mais profondément uni (Chanet, 1996; Dancel, 1999; Thiesse, 1997).

Entre 1969 et 1980, l'histoire, la géographie et l'éducation civique, ont été regroupées dans un vaste ensemble dénommé " activités d'éveil » (avec les sciences expérimentales, les disciplines artistiques et manuelles). Leur contenu est resté sans programme précis pendant toute cette période et, de fait, elles étaient moins enseignées qu’auparavant (Girault, 1983). À partir des années 1980, elles sont restaurées comme enseignements

5 Pour ce qui concerne l'éducation civique, elle est revalorisée dans le cursus scolaire par Jean-Pierre Chevènement en 1985. Depuis, sa place dans les enseignements de l'école élémentaire a constamment été mise en avant. 
spécifiques à l'école élémentaire, dotés d'horaires et de programmes spécifiques $^{5}$. Le thème de l'Europe fait son entrée dans le curriculum scolaire à cette époque, dans les trois disciplines. Mais dans le cadre de programmes traitant essentiellement de la France, il demeure très périphérique.

Une dizaine d'années plus tard, alors que l'idée de citoyenneté européenne vient d'être consacrée par les institutions communautaires, la situation a peu évolué. Les programmes adoptés en 1995 mentionnent "la construction européenne» ou "l'Union européenne" (MEN, 1995, 6870) sans les décrire davantage et préciser leur sens. Compte tenu de leur caractère sommaire (à l'image de la plupart des programmes scolaires), cela n'est pas surprenant; néanmoins, leur contenu indique assez clairement que l'UE n'y est pas conçue comme un sujet majeur et qu'elle n'est pas présentée comme une nouvelle communauté politique. Une place prépondérante continue d'être accordée à l'étude de la France en histoire et en géographie. En outre, dans le programme de géographie, l'UE n'est pas privilégiée par rapport à l'étude du continent européen dans son ensemble. L'UE est aussi strictement arrimée au principe national. Les intitulés des sections où le thème est présent - "Le XXe siècle (1914-19...) : la France dans un monde bouleversé " pour l'histoire (MEN, 1995, 68) et "La France en Europe " pour la géographie (MEN, 1995, 70) - témoignent en effet d'un regard qui reste franco-centré. En géographie, il s'agit de "montrer [l'] intégration [de la France] dans l'Union européenne ». C'est la France qui appartient à l'UE: les Européens comme co-citoyens ne constituent pas un groupe identifiable. Les élèves doivent étudier "la carte de l'Europe ", savoir délimiter "l'Union européenne " et identifier les "États européens" (MEN, 1995, 70). L'UE (comme le continent européen) est ainsi étudiée à travers ses États, dans une conception qui reste centrée sur l'État nation. Elle n'apparaît guère comme une nouvelle forme de communauté politique: le thème est singulièrement absent du programme d'éducation civique.

Les programmes scolaires publiés quelques années plus tard, en 2002, marquent une certaine évolution. L'une des nouveautés de ces programmes est de faire une place plus explicite et plus forte qu'auparavant à une dimension européenne et mondiale. Cela se marque notamment dans les intitulés de certaines sections des programmes, qui cessent de faire référence à la France: en histoire, le "XX" siècle et le monde actuel " (MEN, 2002a, 214) remplace "la France au XXe siècle "; en 
géographie une section s'intitule "Espaces européens, une diversité de paysages" (MEN, 2002a, 214), au lieu de "la France en Europe", titre utilisé en 1995. En continuité avec ces intentions globales, l'Union européenne acquiert plus de place dans les contenus d'enseignement: son étude est réintroduite en éducation civique; elle est accrue et précisée en géographie.

Néanmoins, des continuités importantes existent entre ces textes récents et les programmes scolaires antérieurs. En effet, si la place de l'Union européenne dans les programmes s'accroît, elle reste limitée. Aucune grande section ne lui est spécifiquement consacrée. En géographie, le thème demeure traité parallèlement à l'ensemble du continent européen. L'étude de l'UE doit en outre rester "sommaire ".

L'Union européenne continue également d'apparaître dans les textes de 2002 comme un groupe d'États, dont le sens demeure indéfini. La brièveté qui caractérise la manière dont sont habituellement décrits les sujets d'étude dans les programmes scolaires n'est pas seule en cause. Les programmes de 2002 sont plus détaillés que les précédents et ils sont aussi accompagnés de documents d'application en histoire et en géographie. Or ces derniers ne donnent pas d'indications plus précises sur la nature et le sens de l'UE: ils la définissent comme un " projet économique et géopolitique ", sans plus de commentaires (MEN, 2002b, 27). À la fin de la présentation de chaque discipline, les programmes identifient dans un encadré synthétique ce que l'élève doit "avoir compris et retenu ": pour ce qui est de l'Union européenne, l'élève est censé avoir retenu de ses leçons de géographie «les États qui $[\mathrm{y}]$ participent" (MEN, 2002a, 222).

Ces caractéristiques sont dans une certaine mesure à relier à la nature de la géographie scolaire, depuis longtemps dominée par l'étude des paysages et par l'identification des grands ensembles et des grandes divisions géographiques, notamment des États. À travers l'enseignement géographique, l'UE peut donc difficilement être abordée autrement qu’à partir des États. Mais ces orientations se retrouvent également dans le programme d'éducation civique. Celui-ci fait également de l'UE un groupe de pays sans signification spécifique. L'élève doit adopter une attitude positive à l'égard de ces pays: le désir de mieux les connaître. Il est en effet indiqué que les enseignants "développent la curiosité de leurs élèves sur les pays de l'Union européenne dans les séquences de géographie et dans celles consacrées à l'apprentissage d'une langue étrangère " (MEN, 2002a, 180). 
Europe et histoire: un discours scolaire contradictoire

Un autre élément des textes de 2002 contribue à priver l'Union européenne de consistance propre et de signification: son retrait de l'enseignement de l'histoire. Les textes contiennent quelques références à la construction européenne, mais renvoient son étude à la géographie. Les documents d'application identifient, pour chaque période historique, un petit nombre de "repères chronologiques" et de "personnages et groupes significatifs"; ceux qui sont indiqués en gras sont considérés comme indispensables. La "mise en marche de l'Europe " en 1957 et la " création de l'euro" en 2002 font partie des dates qui sont identifiées, mais elles sont « facultatives " (MEN, 2002b, 16). Les fondateurs de l'Europe, les figures politiques majeures qui ont ensuite contribué à l'intégration européenne ne sont pas cités dans la liste de personnages, même comme repères non indispensables. Les rédacteurs des programmes ont peut-être voulu éviter les redondances entre l'histoire et la géographie, mais l'absence de l'Union européenne dans le programme d'histoire n'est pas sans conséquences. Cela ne favorise pas son appréhension comme projet politique et en fait une réalité largement désincarnée, privée des référents symboliques forts (évènements et personnages historiques) qui donnent corps à une collectivité.

Les rédacteurs des programmes semblent cependant s'employer à fonder une histoire européenne pour les périodes antérieures à la construction européenne. En effet, la France n'est plus la seule entité du récit historique scolaire. Pour certains phénomènes historiques (tels que le développement du christianisme au Moyen Âge, les idées nouvelles de la Renaissance, les grandes découvertes et l'esclavage aux Temps Modernes, le développement industriel et urbain, la colonisation et les aspirations démocratiques au XIX ${ }^{\mathrm{e}}$ siècle), ce sont l'Europe et les Européens qui deviennent le cadre et les acteurs de l'histoire (MEN, 2002b, 11-15). L'intention des rédacteurs de ces textes est-elle d'encourager chez les élèves l'idée d'une certaine unité culturelle européenne, fruit de l'histoire, dans le but de favoriser aujourd'hui un sentiment d'appartenance à l'Union européenne?

Si tel est le cas, l'entreprise manque largement de cohérence interne puisque ce discours d'unification par l'histoire s'arrête au seuil du $\mathrm{XX}$ siècle. Il n'est pas relayé par le récit de l'unification politique du continent à travers l'histoire de la construction européenne. Au contraire, 
l'accent est mis pour l'histoire proche sur la division de l'Europe: les deux conflits mondiaux et "l'extermination des juifs par les nazis" font en effet l'objet d'une étude privilégiée pour le XXe siècle (MEN, 2002a, 214215). Le mode de présentation du siècle dernier pourrait alors contribuer à nourrir chez les enfants des affects négatifs envers certains pays européens, tout particulièrement l'Allemagne ${ }^{6}$.

Par ailleurs, les éléments d'unité historique et culturelle mis en relief dans les programmes scolaires français entrent dans une certaine mesure en contradiction avec la devise officielle de l'UE - " Unie dans la diversité "- et avec ses frontières actuelles. Par les références à la Renaissance, au christianisme et aux cathédrales du Moyen Âge (sans mention de la religion orthodoxe), à la révolution industrielle, à la démocratie et à la colonisation au XIXe siècle, l'histoire commune ainsi tracée concerne pour l'essentiel l'Europe occidentale.

\section{Une collectivité incertaine}

Au total, telle qu'elle apparaît dans les programmes scolaires, l'évocation de l'Union européenne paraît peu propice à la construction d'un sentiment d'appartenance européenne. L'identité européenne a souvent été décrite comme étant principalement fondée sur une projection vers le futur plus que sur le sentiment de partager une histoire commune singulière ${ }^{7}$. Toutefois, en n'attribuant aucun sens particulier à la construction européenne, fut-il flou, indécis, multiple, les programmes ne contribuent pas à encourager cette orientation des enfants vers un futur européen.

La formation d'un "nous" ne requiert-elle pas aussi " un environnement socialisé, caractérisé par ses gens, une convivialité et un univers de quotidienneté" ? (Baugnet, 2006). Parce que l'Union européenne apparaît avant tout dans les programmes de 2002 comme une collection d'États et que ses grandes figures politiques ne sont pas évoquées, les " gens » de l'Europe sont singulièrement en retrait dans cette image.

Le curriculum trace toutefois un chemin possible vers une forme de " convivialité européenne »: outre la curiosité pour les pays de l'UE, le

\footnotetext{
6 Dans son étude des représentations de jeunes enfants français, Katharine Throssell signale d'ailleurs la persistance des associations de l'Allemagne à son passé nazi (Throssell, 2007).

7 Ce lien avec le futur apparaît notamment dans les perceptions des citoyens (Belot, 2000) ou dans les représentations des fonctionnaires européens (Abélès et Bellier, 1996).
} 
programme d'éducation civique préconise de développer les « contacts directs (par correspondance ou courrier électronique) avec d'autres classes d'enfants européens" (MEN, 2002a, 180). On peut s'interroger sur le rôle de ce type de liens horizontaux dans la construction d'un "nous" européen. Si ces échanges accrus peuvent aboutir à une représentation des Européens plus affective et plus concrète, les assimiler à des " proches " par le biais des relations vécues, ils ne semblent toutefois pas suffisants. Le sentiment d'appartenance à une communauté politique ne découle-t-il pas, en suivant David Easton $(1965,185)$, du sentiment de faire partie d'un groupe spécifique, différent des autres et partageant un destin politique commun? C'est la question du lien spécifique entre Européens - par rapport aux relations susceptibles d'exister par ailleurs avec d'autres pays du monde - qui se trouve posée ${ }^{8}$.

\section{Entre la nation et l'humanité universelles, une Europe sans relief}

Le faible relief pris par l'Union européenne dans l'enseignement élémentaire nous semble devoir être rattaché à une conception de la mission civique de l'école qui reste ancrée dans le couple nationuniversel, couple dont une partie de la signification a aujourd'hui évolué. Dans un contexte actuel de mondialisation, une alternative est en théorie ouverte: soit on dépasse la nation comme cadre prévalent de la citoyenneté et de l'appartenance communes, soit on tente de la concilier avec l'universel. C'est cette deuxième option qui semble choisie par les concepteurs des programmes de 2002.

À côté des aperçus sur des phénomènes historiques européens et mondiaux, le récit scolaire de l'histoire, reste, à l'école primaire, largement national. Les auteurs des programmes d'histoire s'emploient à ancrer la nation dans les repères des enfants: parmi la liste de dates et de personnages, ceux à retenir impérativement sont quasi-exclusivement

\footnotetext{
8 La problématique des relations horizontales et affectives entre Européens a été abordée lors du deuxième colloque international de la Section d'études européennes de l'Association française de science politique, intitulé "Amours et désamours entre Européens. Pour une sociologie politique des sentiments dans l'intégration européenne ". Les communications présentées lors de ce colloque soulignaient dans l'ensemble la faiblesse d'un sentiment de communauté entre Européens (voir les publications d'une partie d'entre elles dans Politique européenne (2008), $\mathrm{n}^{\circ} 26 / 3$, "Amours et désamours entre Européens. Vers une communauté européenne de citoyens?»).
} 
nationaux. Comme dans les manuels scolaires du secondaire (Schissler et Soysal, 2005), ces événements et ces figures sont largement démythifiés, le ton employé pour les décrire ne dénote aucune coloration affective ou élogieuse. Le récit historique scolaire n'épargne d'ailleurs pas en 2002 le " mythe national " (Citron, 2008) en soulignant les oppressions dont s'est rendu coupable l'État français, même sous sa forme républicaine, à travers l'histoire, à l'intérieur comme à l'extérieur de son territoire9. En bref, l'histoire scolaire n'évoque plus seulement la France et ne le fait plus dans les mêmes termes, mais elle reste une histoire où le national reste l'angle de vue et le support principaux.

Au-delà de l'épaisseur des siècles, c'est la dimension universaliste de la nation qui est particulièrement mise en avant. Le programme d'éducation civique, par exemple, associe fortement la France aux principes universels de démocratie et des droits de l'homme: "Etre citoyen en France " (titre de la troisième section du programme) revient principalement à découvrir une histoire française qui est celle de l'affirmation progressive de ces principes (MEN, 2002a, 179-180). La participation à la vie démocratique et la défense de l'idéal des droits de l'homme sont ensuite suggérées comme des activités civiques positives. Il s'agit donc en éducation civique de susciter l'attachement de l'enfant à des principes politiques qui ne sont pas désincarnés et qui ne sont pas rattachés à l'Europe, mais à la nation. Cette image universaliste de la nation n'est pas nouvelle: elle a caractérisé le discours scolaire depuis la Troisième République (Déloye, 1994,111-118; Lelièvre, 2001).

Les textes de 2002 mettent aussi en avant, plus en filigrane, une deuxième figure de l'universalisme, qui se rapporte cette fois à l'humanité entière et qui est plus contemporaine car elle répond aux évolutions du contexte international. Cela est particulièrement repérable en histoire et en éducation civique. En histoire, la description générale du xxe siècle donne lieu à un commentaire où l'unification européenne acquiert un sens positif de pacification et de réduction des inégalités entre régions du monde (MEN, 2002b, 16). Toutefois, l'UE perd ici sa particularité car elle est rapprochée dans ce passage de l'action d'organisations internationales telles que l'ONU. En éducation civique, l'UE est abordée dans la dernière section du programme intitulée « S'intégrer à l'Europe,

9 À travers, par exemple, le traitement des thèmes de la collaboration sous le régime de Vichy, de l'esclavage, ou de la colonisation. 
découvrir la francophonie, s'ouvrir au monde " (MEN, 2002a, 180). À travers cet intitulé, l'Europe semble acquérir un statut privilégié : le terme employé ("sintégrer») est révélateur d'une logique plus inclusive. Néanmoins, en étant traitée parallèlement à d'autres ensembles supranationaux, la spécificité de l'UE reste faible. Le contenu de la section montre d'ailleurs qu'elle constitue un collectif incertain, alors que la francophonie et le monde représentent des entités plus identifiables. $\mathrm{Ne}$ sont évoqués pour l'Europe que les pays qui la composent. La francophonie est quant à elle "une communauté de langues et de cultures". Concernant le monde, les auteurs des programmes évoquent différents éléments qui en font une collectivité potentielle: le partage de problèmes communs (" économiques et culturels », «l'environnement»), les droits humains (à travers l'évocation des droits de l'enfant), et les « solidarités nécessaires " du fait des inégalités entre régions du monde. Le programme d'éducation civique suggère ainsi une nouvelle identité civique pour le futur citoyen, élargie au monde entier, et certaines pratiques civiques associées, telles que le respect de l'environnement, la défense des droits de l'homme ou l'implication en faveur de l'aide aux pays les plus pauvres.

Lécole doit donc initier l'élève à deux collectifs privilégiés: la nation et l'humanité entière. Entre ces deux figures de l'universel, cadres potentiels d'un engagement civique identifiable, l'Europe est absente. Seul le national est cependant véritablement associé au vocabulaire de l'identité et de l'appartenance: à la nation seulement on "appartient »; à l'égard du monde, on doit se sentir "solidaire " ou on doit "s'ouvrir " (MEN, 2002a, 179-181).

Au total, l'enseignement de l'Europe est donc largement freiné par un langage de l'universel qui s'étend au monde entier, et plus fondamentalement, par la primauté qui reste accordée au national dans les repères identitaires et affectifs des enfants. Que l'école primaire doive avant tout construire l'appartenance à la nation n'a jamais été véritablement contesté dans les débats publics sur l'école, et n’a jamais été remis en cause par les autorités éducatives. Mission principale de l'école primaire de la Troisième République, la formation d'une conscience nationale l'est restée avec le développement de l'enseignement secondaire comme enseignement de masse. Un partage des rôles a été instauré et a perduré au fil des décennies: au primaire le national, au secondaire une ouverture 
plus importante à l'Europe et au monde ${ }^{10}$. Les principaux acteurs dans l'élaboration du programme de 2002 n'ont pas rompu cette tradition, bien au contraire. Les programmes de 2002 ont été rédigés par un groupe d'experts, dirigé par Philippe Joutard, historien et recteur d'Académie, en liaison avec le bureau des écoles et le Conseil national des programmes. Dans une interview accordée aux Cahiers d'histoire en 2004, Ph. Joutard affirme explicitement que la construction de l'identification à la nation à travers l'enseignement primaire a représenté un objectif majeur et a fait l'objet d'un consensus parmi les membres du groupe d'experts. L'Union européenne n'est guère évoquée dans cette interview (Pingué et Joutard, 2004). Le privilège du national dans les programmes du primaire repose ainsi sur une volonté politique consciente.

Au-delà du poids du national, un autre élément pourrait jouer un rôle pour rendre compte du faible relief de l'Union européenne dans les programmes scolaires: les acteurs du ministère les plus acquis à la cause européenne pourraient redouter les réactions négatives des enseignants face à une politique volontariste et clairement affichée de promotion de l'Union européenne par l'école. L'organisation en 2005 de journées d'étude intitulées « "Faire " des Européens? L'Europe dans l'enseignement de l'histoire, de la géographie et de l'éducation civique " à l'initiative de l'Inspection générale de l'Éducation nationale et du Centre d'histoire de Sciences Po a suscité, selon un des participants au colloque, des courriers de protestation. Les organisateurs y étaient accusés de vouloir transformer les enseignants en "petits télégraphistes de Maastricht " (Bergounioux et al., 2006). Les fondateurs de la Troisième République pouvaient compter sur des instituteurs largement acquis à la cause de la nation républicaine; aujourd'hui, les acteurs de l'Éducation nationale savent sans doute que l'Union européenne telle qu'elle se construit fait l'objet de désaccords importants dans la profession.

10 Si la part consacrée à l'Europe dans les programmes du secondaire est sensiblement plus importante que dans le primaire, deux études montrent cependant qu’à ce niveau aussi, le poids du national est prégnant. Le contenu des programmes et des manuels scolaires du secondaire (en histoire, géographie et éducation civique) fait apparaître une oscillation entre une projection positive sur la construction européenne, pensée comme un miroir de l'État nation, et un refus de concurrencer la primauté de la nation (Baeyens, 2000). Une analyse fondée sur une approche de politiques publiques montre que la promotion de la citoyenneté européenne à travers l'enseignement civique du secondaire se heurte en France à la fois à des obstacles institutionnels et à la permanence des idiomes nationaux de la citoyenneté (Girod, 2006). 


\section{L'Europe enseignée : entre différenciation et convergence relative des pratiques d'une classe à l'autre}

Comment les instituteurs se saisissent-ils de ces instructions officielles? Enseignent-ils l'Union européenne et comment?

L'enquête dans les écoles a reposé sur des entretiens, conduits entre 2004 et 2006; le thème de l'Europe y était abordé, mais n'était pas central dans le questionnement ${ }^{11}$. L'enquête comprenait également des observations dans les classes, mais nous n'avons pas eu l'occasion d'observer des séances consacrées à l'Union européenne ${ }^{12}$. Nos informations proviennent essentiellement sur ce point des pratiques rapportées par les enseignants lors des entretiens et du contenu des cahiers d'élèves en notre possession ${ }^{13}$.

\section{Des pratiques diverses}

Les pratiques d'enseignement ayant trait à l'Union européenne sont diverses. On peut relever trois types de pratiques, qui se différencient en

\footnotetext{
11 Les entretiens ont été conduits auprès d'une trentaine d'instituteurs, différenciés en termes d'âge, de sexe, de type d'entrée dans le métier, de rapport à la religion, d'origines sociales, d'opinions politiques, etc. L'entretien était constitué de deux parties. Dans un premier temps, les interviewés devaient réagir à des rédactions qui avaient été écrites en classe par leurs élèves à l'occasion de l'enquête, à partir d'une consigne ("dire qui ils sont en parlant des groupes auxquels ils appartiennent ") et d'une liste d'exemples qui était commentée par l'enseignant lui-même. Cette liste comprenait des références à différents groupes (la religion, la classe sociale, l'appartenance ethnique, le quartier, la ville, la France, être " citoyen du monde ", etc.). Un item sur l'Europe était inclus: « je suis citoyen de l'Union européenne ». Dans un second temps de l'entretien, les instituteurs répondaient à une série de questions sur leurs pratiques et leurs représentations (concernant notamment l'éducation à la citoyenneté, les contenus en histoire et en géographie, etc). Une question formulée de manière très générale portait sur l'Europe (si le thème n'avait pas été évoqué spontanément précédemment) : il s'agissait de demander à l'enseignant s'il parlait de l'Europe aux enfants et s'il jugeait cela important. 12 Non seulement parce que la question de l'enseignement de l'Europe n'était pas au cœur de la recherche, mais surtout parce que de manière générale, il était difficile d'être présent au "bon moment ", c'est-à-dire lorsque les pratiques d'enseignement et les comportements les plus significatifs d'un point de vue civique et politique étaient observables. La plus grande partie du temps en classe est consacrée à des apprentissages instrumentaux, en français et en mathématiques.

13 Nous avons pu collecter les cahiers d'élèves de cinq classes de notre enquête. Une quinzaine de cahiers d'élèves ont été collectés par ailleurs, hors du cadre de l'enquête de terrain; dans ce cas, le profil des enseignants n'est pas connu. L'homogénéité de contenu que l'ensemble de ces cahiers révèle sur certains points permet de tirer quelques conclusions.
} 
fonction de la place quantitative qui est accordée au thème dans l'enseignement, mais aussi du mode de présentation de l'UE.

Dans une large mesure, cette diversité n'est pas liée au type de public scolaire, à sa composition sociale et ethnique: les classes où l'Union européenne a constitué un sujet d'enseignement important, présenté de manière largement positive, sont situées dans des écoles de zone d'éducation prioritaire (ZEP) comprenant une forte proportion d'enfants de parents immigrés, ou dans des écoles localisées dans des quartiers plus favorisés et moins mixtes d'un point de vue ethnique. La variété des pratiques n'est pas non plus à rapporter à l'âge de l'enseignant, ni à son parcours professionnel (passage par l'école normale, par l'IUFM ou titularisation liée à l'expérience, expérience dans un autre domaine que l'enseignement).

Dans certaines classes, l'Union européenne a été abordée en détail et décrite de manière très positive. C'est le cas par exemple de la classe de Monique. Âgée de 42 ans, Monique travaille dans une école mixte d'un point de vue social et ethnique, située dans un village à proximité de Nice. Elle est devenue enseignante il y a quatre ans, après une expérience dans le secteur privé, dans le domaine du marketing. Dans le cahier d'élève issu de sa classe de CM2, l'UE occupe la plus grande part des travaux de géographie. Elle est associée à de multiples significations positives: au-delà de la création d'un espace sans frontières, sont mis en avant, par les documents que l'enseignante a choisis et fait lire à ses élèves, la démocratie, la paix, la modernisation économique, la solidarité en faveur des pays et des régions les moins développés, la lutte contre les inégalités sociales et la protection de l'environnement. Les institutions européennes sont également rapidement présentées à travers un texte qui les décrit de manière très simplifiée. En éducation civique, Monique a évoqué le référendum sur le traité constitutionnel qui devait avoir lieu cette année-là. Un document photocopié traitant de la Constitution est inclus dans le cahier. Il la présente de manière particulièrement dépolitisée: la raison d'être de la Constitution est d'adapter les règles de fonctionnement de l'UE à une Europe qui comprend désormais vingt-cinq membres; rien n'évoque le débat en cours sur le contenu de la Constitution. Les élèves de la classe de Monique ont également dû conduire une recherche exhaustive sur un des pays membres de l'UE: présenter sa capitale, sa (ses) langue(s), ses religions, certaines de ses caractéristiques politiques et culturelles. Une rédaction rédigée par l'élève 
conclut le cahier de géographie, avec pour sujet "Qu'as-tu retenu de ton cours sur l'UE?». L'élève a bien retenu ses leçons: il énumère en effet les divers bienfaits de l'Europe, en particulier le fait de ne pas "payer la douane", "[d']aider les pays les plus pauvres", " [d']éviter la guerre » et de construire la "fusée Ariane ". Il termine sa rédaction par "moi je trouve que c'est bien l'Union européene (sic) ", et récolte un " $A$ » avec la mention "Très bien ". La manière de présenter l'Europe en classe semble donc avoir été chargée d'affects et de tonalité positive dans certaines classes.

Dans d'autres classes, l'UE a aussi fait l'objet d'une description relativement détaillée, mais le traitement du sujet a été plus factuel et moins coloré positivement. Les élèves ont dû apprendre les différentes étapes de la construction européenne, les pays membres de l'UE et de la zone euro, le nom et la principale fonction des institutions politiques. Dans ces classes, l'UE apparaît principalement comme une réalité économique. Ce type de présentation se retrouve par exemple dans la classe de Gaëlle, âgée de 31 ans, qui enseigne à Brest en CM2, dans une école de ZEP comprenant un tiers d'enfants de parents immigrés. Le contenu des documents et des exercices inclus dans le cahier de ses élèves privilégie la dimension économique: la "puissance économique " de l'UE est donnée à voir, la liberté de circulation des hommes et des marchandises et les projets économiques européens (à travers l'exemple d'Airbus) sont évoqués.

Dans d'autres classes encore, situées dans des écoles au public différencié, les travaux sur l'Union européenne ont été beaucoup moins développés, voire inexistants. Une seule page évoque le sujet dans les cahiers. Elle comprend une carte avec les pays membres de l'UE et une courte chronologie de la construction européenne.

À côté de ces enseignements, certains instituteurs se sont également investis dans des projets spécifiques. C'est le cas d'Olivier et de Patrick, qui travaillent dans la même école de ZEP à Brest et qui enseignent actuellement en CE2-CM1 et en CM2 respectivement. Olivier, 33 ans, est professeur des écoles depuis quelques années, et Patrick, 52 ans, fait partie de l'Éducation nationale depuis presque vingt ans. Tous deux ont décidé l'an dernier de participer à un programme Comenius, avec d'autres collègues motivés par un projet de ce type. Ils ont ainsi été amenés à échanger avec leurs collègues européens, et à effectuer des visites dans les établissements partenaires (en Suède, en Italie et en Angleterre). Des correspondances scolaires entre élèves ont également été mises en place. À travers ce projet, c'est essentiellement une Europe des 
différences nationales qui est donnée à voir aux élèves. Il s'agit de faire découvrir aux enfants que dans d'autres pays "c'est différent" sans que cela soit "moins bien". L'Europe est ici une des voies pour faire comprendre cette relativité. Olivier explique par exemple que les fêtes ont été le thème des échanges entre les élèves cette année-là: cela a permis à ses élèves de prendre conscience de la diversité des pratiques culturelles, selon les pays, relatives notamment aux fêtes de Noël. Les enfants ont échangé des colis contenant divers objets typiques de ces fêtes dans leur pays. Des " colis lexique » étaient également envoyés avec des mots de vocabulaire dans la langue nationale sur différents thèmes (jours de la semaine, par exemple) et leur traduction en anglais. Pour Olivier, le projet Comenius permet aux élèves de connaître les spécificités de chacun des pays, de "voir ce qui va bien » et "moins bien " en France et ailleurs. Patrick évoque également cette diversité culturelle et mentionne, en plus du projet Comenius, le choix de romans anglais, suédois et italiens en littérature. Monique a elle aussi mis en place des correspondances entre élèves, mais dans un cadre plus informel: les enfants de sa classe correspondent régulièrement avec des élèves issus de trois autres pays européens (la Pologne, l'Allemagne et l'Espagne). L'enseignante explique aussi que ces échanges ont été l'occasion de "comparer» les pays entre eux, en particulier en termes de niveau de vie, d'organisation de la scolarité, de modes de vie.

Dans certaines classes, l'Union européenne acquiert donc davantage de sens: certains enseignants présentent en effet l'UE de manière positive en y associant différentes références, en particulier à la démocratie, à la paix et à la solidarité économique. Dans d'autres classes, l'UE apparaît comme un groupe de pays dont le sens n'est pas donné, ou dont la principale signification est d'être avantageux sur le plan économique.

Dans les classes où existent des correspondances scolaires avec d'autres établissements en Europe, les élèves sont davantage sensibilisés à la diversité culturelle européenne: ils acquièrent une connaissance plus précise et aussi plus concrète de différents pays européens à travers des relations humaines. Ces échanges peuvent aussi les conduire à ressentir un sentiment de proximité plus fort vis-à-vis des enfants issus d'autres pays européens. Pour autant, cette mise en contact privilégiée avec des pays et des enfants européens a aussi un statut fragile: une des significations principales données à ces projets étant la sensibilisation des élèves aux différences culturelles, ces activités peuvent aussi concerner d'autres pays 
du monde. Par exemple, les correspondances scolaires avec trois pays européens mises en place par Monique et considérées par elle comme un des points clefs de son enseignement de l'Union européenne concerneront l'année suivante la Réunion et l'Australie, parce qu'elle en a l'occasion. Les activités scolaires sont en partie affaire d'opportunité, et le privilège de l'Europe dans ces relations horizontales créées entre enfants peut se défaire d'une année à l'autre. Dans le même sens, Patrick décrit le projet Comenius en parlant d'une ouverture à l'Europe mais aussi au monde en général. Après avoir mentionné les romans européens qu’il donne à lire à ses élèves, il précise qu'il aimerait bien leur faire lire aussi des "romans sud-américains". La spécificité de l'Europe comme incarnation privilégiée de la diversité culturelle et de l'ouverture à l'autre est donc faible.

\section{Une convergence relative: le primat du national}

D'une classe à l'autre, il existe donc des différences de pratiques importantes concernant l'enseignement de l'Union européenne. On observe toutefois aussi une certaine convergence. Lapproche par les pays qui composent l'Union européenne est en effet le point commun qui se retrouve dans toutes les classes. Dans tous les cahiers où le thème de l'UE est traité, une carte permet de localiser et de nommer les différents pays membres, et, parfois, d'identifier leur drapeau. Dans la très grande majorité des cas, les cahiers comprennent aussi un tableau indiquant la superficie, la population, la capitale, et quelquefois le niveau de richesse des pays de l'UE. L'enfant est ensuite invité à comparer les différents pays entre eux sur ces caractéristiques ou à identifier le pays le plus peuplé/le plus petit, etc. Le travail personnel de recherche effectué par chaque élève sur un pays membre dans la classe de Monique a également été réalisé dans plusieurs classes de l'enquête.

Enseigner l'Union européenne consiste donc, dans une large mesure, à faire mieux connaître ses différents pays. La présentation des étapes clés de la construction européenne va aussi en grande partie dans le même sens d'une approche par les pays: elle commence certes par la CECA et/ou le Traité de Rome, mais la suite de l'histoire est centrée sur les inclusions successives de différents pays. L'Union européenne, à travers toutes ces pratiques, apparaît donc comme un ensemble de nations.

Un autre point commun est la prédominance de la nation française dans le récit historique scolaire et dans la présentation qui est faite de la 
démocratie. L'histoire que les instituteurs enseignent reste essentiellement nationale. Le changement de point de vue apporté par les programmes de 2002 pour certains phénomènes historiques, consistant à centrer désormais le récit sur l'Europe et les Européens, est passé largement inaperçu des instituteurs. Sur ce point, l'histoire enseignée est identique d'une classe à l'autre et ressemble largement à ce qu'elle fut trente ans auparavant ${ }^{14}$ : en dehors des périodes des "grandes découvertes ", de la Renaissance et des deux guerres mondiales (pour lesquelles les figures et les événements évoqués sont européens, ou mondiaux), le cadre de l'histoire est strictement hexagonal. Les instituteurs de l'enquête ancrent leur récit du passé dans le territoire de la Gaule puis de la France, ce qui donne l'image d'une continuité de la nation dans le temps et la dote d'une forte épaisseur historique et culturelle. La vision de la démocratie et des droits de l'homme qui se dégage des pratiques d'enseignement en histoire et en éducation civique est également associée en grande partie à la nation. L'éducation civique dispensée par une grande partie des instituteurs de l'enquête met en relief les droits de l'homme et de l'enfant, mais cet apprentissage n'est pas déconnecté de la France du fait de l'importance prise, dans leurs pratiques d'enseignement, par la présentation de la Déclaration des droits de l'homme française. En outre, seules les institutions politiques nationales sont abordées de manière relativement détaillée dans certaines classes. La responsabilité du citoyen est mise en valeur à ce niveau, le vote et les élections étant systématiquement évoqués. La présentation des institutions européennes est au contraire très sommaire et centrée sur le rôle respectif des différentes institutions et leur localisation dans les «capitales » de l'Europe. Le citoyen européen a très peu de place dans cette image qui est donnée de l'UE; il n'est apparu dans l'enseignement que de manière exceptionnelle, parce que le référendum sur le traité constitutionnel était organisé cette année-là. C'est donc la France qui continue d'incarner en premier lieu la démocratie et les droits de l'homme. Au total, l'appartenance nationale continue ainsi d'être transmise à travers tout un ensemble de contenus, au-delà de la seule langue, où la France reste l'entité collective essentielle.

14 Comme le montre la comparaison entre les cahiers d'élèves actuels et les quelques cahiers d'élèves des années 1980 en notre possession. 
Rendre compte des pratiques: attitudes envers l'Europe, poids des instructions officielles et des routines professionnelles

Le poids des orientations et des idéologies politiques: les logiques à l'œuvre dans la critique ou la valorisation de l'UE

Les pratiques d'enseignement relatives à l'Union européenne paraissent en partie liées aux attitudes des instituteurs à l'égard de l'Europe. Elles mettent ainsi en jeu leurs orientations et leurs représentations politiques. Les entretiens ont été conduits à des moments où l'actualité européenne était particulièrement vive: en particulier, une dizaine d'entretiens ont été menés au printemps 2005, dans le contexte du référendum européen, et une dizaine un an après, au printemps 2006. Dans le discours de plusieurs instituteurs, ce contexte se fait directement sentir, les interviewés exprimant leur opposition ou leur approbation à l'égard du référendum, et plus largement, de l'UE telle qu'elle se construit.

Ces clivages sur le référendum de 2005 ne sont pas surprenants. Les syndicats enseignants ont été partagés sur ce vote: Sud-Éducation appelait à voter " non ${ }^{15}$; le syndicat majoritaire chez les instituteurs, le SNUIPP (rattaché à la FSU) rejetait le traité sans donner de consigne de vote ${ }^{16}$, tandis que le SE-UNSA ${ }^{17}$ et le SGEN-CFDT ${ }^{18}$ exprimaient des réserves sur le texte mais, le considérant comme une étape nécessaire, défendaient une adhésion à celui-ci. Malgré ces différences de positionnement, les syndicats se retrouvaient sur la défense d'une meilleure prise en compte dans le traité de la dimension sociale de l'Europe. Au-delà des syndicats, les sondages d'opinion disponibles montrent que les enseignants - tous niveaux d'enseignement confondus ${ }^{19}$ - se distinguent par

15 Voir la prise de position exprimée le 25 mars 2005 sur le site web du syndicat: http://www.sudeducation.org/article292.htlm

16 Voir le texte du 20 mai 2005 publié sur le site web du syndicat et présentant les positions du SNUIPP et de la FSU: http://www.snuipp.fr/spip.php?article2435.

17 Voir l'éditorial de Luc Bérille, secrétaire général du SE-UNSA, dans la revue du syndicat, L'Enseignant, ${ }^{\circ}$ 84, mai 2005.

18 Voir la prise de position de Jean-Luc Villeneuve, secrétaire général du SGEN, dans la revue Profession éducation, ${ }^{\circ} 146$, avril 2005.

19 Ces données sont tirées de l'enquête post-électorale présidentielle 2007 réalisée par l'IFOP pour le Cevipof avec le soutien du ministère de l'Intérieur (CEVIPOF/ministère de l'Intérieur, 2007). La catégorie des instituteurs n'est pas isolée du reste du monde enseignant. 
un niveau d'adhésion à la construction européenne considérablement élevé ${ }^{20}$. Dans le même temps, une forte majorité d'entre eux (63\%) expriment une crainte à l'égard de la menace que pourrait faire peser la construction européenne sur la protection sociale en France ${ }^{21}$. L'orientation du vote enseignant sur le référendum de 2005 témoigne d'oppositions internes à la profession $(\mathrm{CEVIPOF} /$ ministère de l'Intérieur, 2007, vol. 2, p. 205) : une courte majorité a voté « oui » (46\%, contre $42 \%$ pour le «non »).

Aucun des enseignants de notre enquête n'exprime d'opposition à l'idée générale d'unification européenne, bien au contraire. Ce sont les orientations prises par cette unification qui sont rejetées par certains. Les instituteurs les plus critiques sont ceux qui sont les plus politisés et qui se situent à l'ultra-gauche. Gaëlle, par exemple, a accordé une certaine importance au thème de l'Union européenne dans son enseignement. Mais, comme on l'a vu, sa présentation du thème a été très factuelle et dominée par une approche économique de l'UE. Militante à SudÉducation et dans une association de défense des sans-papiers, Gaëlle fait part en entretien de ses réticences face à l'Union européenne. Elle a voté « non » en 2005 , ne se démarquant pas en cela de la position officielle de son syndicat:

Gaëlle - [En géographie] on a fait le monde. On a commencé par faire l'Europe, ça, ça me...

$Q$ - Oui, justement je voulais te poser la question...

Gaëlle - L'Union européenne, euh... C'était moins mon truc (rire) Faut le faire. C'est compliqué parce que après y a les idées politiques par rapport à l'Union européenne, tu vois, donc forcément on est enseignant avec ce qu'on vit aussi, avec ce qu'on est.

$Q-T u$ veux dire que l'Union européenne t'es pas trop engagée pour?

20 Les enseignants sont $79 \%$ à penser que " le fait pour la France de faire partie de l'Union européenne est une bonne chose ", contre $50 \%$ en moyenne dans l'échantillon global (CEVIPOF/ministère de l'Intérieur, 2007, vol. 1, p. 24).

${ }^{21}$ Les enseignants se situent là à un niveau intermédiaire (63\%), les professions libérales et les cadres supérieurs étant moins nombreux à exprimer cette crainte $(55 \%)$, les artisans, commerçants et chefs d'entreprise encore moins ( $48 \%$ ), par opposition aux employés $(73 \%)$ et aux ouvriers $(74 \%)$. La question précise était la suivante: «Certaines personnes peuvent avoir des craintes concernant la construction européenne. Pour chacun des aspects suivants, dites-moi si vous en avez personnellement peur ou non. Qu'avec la construction européenne, il y ait moins de protection sociale en France " (CEVIPOF/ministère de l'Intérieur, 2007 : vol. 1, p. 26). 
Gaëlle - Je trouve ça vachement bien l'Europe, je trouve ça vachement bien que ça existe, mais enfin l'Europe telle qu'on est en train de la construire, non, j'ai voté "non » au traité de la Constitution européenne. Et ça par contre je veux pas leur dire, je veux pas qu'on leur dise parce que ça, je trouve que ça va trop loin.

Ce type d'attitude critique vis-à-vis de l'Union européenne a certaines conséquences sur la manière dont elle est enseignée. La critique anti-libérale de l'UE favorise un traitement du thème moins positif, moins engagé et plus factuel, les enseignants ayant peur d'exprimer leurs opinions politiques sur le sujet. La dimension économique de l'UE est mise en avant, tandis que les questions sociales et les thèmes touchant à la solidarité sont évités.

À l'inverse, d'autres interviewés évoquent en entretien leur attachement à l'Union européenne, leur vote positif au référendum ou leur déception par rapport au résultat du scrutin. Suite à une question lui demandant pourquoi elle aime tant travailler sur l'Union européenne en classe, Monique, qui dit voter à droite tout en faisant part de sa distance avec la politique, répond d'emblée qu'elle "vote "oui " pour l'Europe " et qu'elle est " très européenne ». Armelle exprime aussi en entretien son attachement à l'Union européenne. Cette enseignante âgée de 43 ans, qui se positionne à gauche de l'échiquier politique, enseigne depuis plus de vingt ans, après être passée par l'école normale. Elle travaille actuellement, comme Martine, dans une école mixte d'un point de vue social et ethnique, située à Paris. Lors de l'entretien, Armelle regrette que l'Europe n'ait pas été un thème de la consigne repris dans les rédactions rédigées par ses élèves de CM2 à l'occasion de l'enquête: "visiblement ils ne se sentent pas européens du tout ", dit-elle, alors qu'elle a beaucoup travaillé sur l'UE en géographie et en éducation civique au dernier trimestre. Interrogée alors sur l'importance de l'enseignement de l'Europe, elle fait allusion, avec une certaine amertume, au résultat du référendum: "[L'Europe], c'est l'avenir, enfin je croyais que c'était l'avenir parce qu'y a des gens qui le pensent pas", dit-elle.

Les instituteurs les plus favorables à l'Union européenne appartiennent à la droite ou à la gauche modérées et à différentes générations. L'Europe est pour eux associée à différents éléments positifs. Dans le discours de Monique, l'Europe est avant tout le cadre de vie futur de ses élèves comme de ses propres enfants, l'environnement socio-économique qui sera le leur une fois adultes: 
Ayant des enfants petits, je me dis que quand ils vont être grands, ils seront bien contents de pouvoir aller faire leurs études où ils veulent, travailler où ils veulent et qu'il faut que les gamins se rendent compte que maintenant on ira en vacances en... je sais pas, en Roumanie, on ira faire ses études en Belgique et puis on se mariera avec une Allemande etc. C'est important qu'ils se rendent compte.

Monique dit avoir constamment cherché à expliquer à ses élèves, lors des séances sur l'UE, que celle-ci serait désormais leur horizon de vie. La diversité culturelle en Europe est également valorisée dans son discours, comme elle l'est dans le discours d'autres enseignants, orientés politiquement à gauche. Ce qui caractérise les propos de ceux-ci est l'accent mis, à travers l'Europe, sur le dépassement des nationalismes et des appartenances à des groupes restreints. Dans l'entretien d'Armelle, par exemple, l'Europe est valorisée comme détachement supplémentaire - implicitement, par rapport à l'abstraction qu’a déjà représenté la nation - de l'individu par rapport à ses appartenances, ethnique ou religieuse. Après avoir évoqué le résultat du référendum de 2005, Armelle poursuit ainsi :

Armelle - Je trouve important que de se définir autrement que par sa communauté, ou bien sa couleur de peau ou bien ses convictions religieuses. $Q$ - Et là c'est vrai, ça ne faisait pas ça...?

Armelle - Oui. La notion de citoyenneté, de citoyenneté européenne, moi elle m'est chère, donc c'est vrai que j'essaie de la faire vivre en classe. Visiblement c'est pas passé. (rire)

Armelle décrit alors longuement et de manière très négative le poids de plus en plus important pris par l'Islam et la couleur de peau dans son école: les enfants des minorités religieuses et raciales s'identifieraient de plus en plus sur la base de ces groupes. L'Europe émerge donc ici comme un groupe légitime parce qu'elle est perçue comme fondée sur un lien non communautaire.

Pour une autre enseignante, Rose, l'Europe représente un dépassement des nationalismes qui ont marqué le XXe siècle, en particulier des antagonismes entre Français et Allemands. Rose, 38 ans, enseigne dans une école de Brest, composée majoritairement de classes moyennes. Originaire du Limousin, elle est devenue professeur des écoles il y a quatre ans, après avoir été maître auxiliaire en anglais et en allemand pendant une dizaine d'années. Les propos qu'elle tient sur l'Europe se chargent d'une intense tonalité affective. Rose a grandi à proximité d'Oradour-sur-Glane; elle 
explique que les sentiments négatifs à l'encontre des Allemands étaient répandus dans son milieu familial et local et que son choix d'étudier la langue allemande au collège a fait l'objet de critiques. Elle a ensuite été confrontée à des préjugés sur les Allemands lorsqu' elle l'a enseignée à des élèves. L'Europe acquiert dans son discours une valeur positive de paix et d'acceptation de l'autre national:

Je trouve que bon il faut qu'ils [les élèves] soient démocrates mais au sens plus large que la France. Européen aussi, ouvert pour éviter tout ça. Pour éviter ça, pour éviter de renouveler tout ça, hein, c'est clair. C'est vraiment... ça c'est clair. Pour avoir justement vu tout ce que la rancœur peut donner.

Pour ces enseignants, apprendre l'Europe aux enfants signifie mettre en relief une appartenance plus large et plus inclusive que les groupes nationaux, ethniques ou religieux. Le thème européen acquiert davantage de place dans leur enseignement que dans les classes d'enseignants plus critiques ou plus indifférents à l'égard de l'Europe. Il est également possible que leur discours positif sur l'UE comme forme de dépassement des frontières entre les groupes ait pu être exprimé en classe, d'une manière ou d'une autre ${ }^{22}$.

Un objet politique qui demeure cependant relativement flou: défense ou critique universaliste de l'Europe et indifférence à son égard

Au-delà de ces différences d'attitudes, l'Europe reste toutefois un objet politique relativement flou. En effet, certains enseignants font preuve d'une méfiance envers l'Europe pour les mêmes raisons qui poussent d'autres enseignants à y être attachés. Ces instituteurs, qui se situent aussi à gauche, se différencient en effet peu des enquêtés décrits précédemment, qui insistent sur l'Europe comme moyen de dépasser des affiliations considérées comme restreintes et intolérantes. C'est le cas de Céline, 29 ans, qui se dit à gauche, et qui effectue sa première année d'enseignement dans une école située dans un quartier très favorisé à Paris, avant de rejoindre une école de ZEP de la banlieue parisienne. Son

\footnotetext{
22 Nous ne sommes toutefois pas en mesure de le confirmer car nous n'avons eu l'occasion d'entendre ce type de commentaires en classe. Les cahiers d'élèves n'en portent pas trace non plus, mais cela est à relier au fait que les écrits scolaires privilégient les connaissances précises et factuelles (même si le choix de ces connaissances et leur mode de présentation ne sont pas neutres).
} 
discours sur l'Europe est empreint d'indifférence, sinon de méfiance. Pendant l'entretien, Céline se réjouit chaque fois qu'elle constate que les rédactions de ses élèves reprennent l'idée de "citoyen du monde " (présente dans la consigne) plutôt que les items sur l'appartenance nationale, ethnique, religieuse ou sociale. Pour elle, cela montre que les enfants se "sentent humains avant tout». Interrogée sur l'Europe, Céline semble la considérer comme une autre forme de frontière, qui ferait barrage au sentiment d'appartenance au monde entier. C'est ce qui nous semble animer son refus d'accorder un privilège à l'enseignement de l'Europe et sa volonté de mettre en avant une dimension mondiale:

$Q$ - C'est important de parler de l'Europe aux enfants?

Céline - Dans les programmes, on doit. Moi je l'ai quasiment pas fait. Pour moi c'est plus important de parler du monde en entier que de l'Europe. Je devrais pas dire ça mais ça me semble pas hyper important. D'ailleurs on sent pas chez eux, comme chez les adultes, une adhésion à l'Europe. On le sent plus dans le monde en entier que dans cette partie de l'Europe. Y'en a qu'un qui en a parlé [dans les rédactions]. Un seul.

L'apprentissage privilégié de l'Europe à l'école présente en fait le risque, pour certains enseignants, de promouvoir une nouvelle forme de fermeture. Le discours de Magali, 30 ans, qui enseigne à Nantes dans une école située en ZEP et qui se positionne à gauche, va dans ce sens. Questionnée sur le rôle de l'école par rapport à l'Europe, Magali fait part de sa méfiance. Le nationalisme belliqueux entretenu par l'institution scolaire dans le passé est mis en parallèle avec l'instrumentalisation éventuelle de l'école actuelle pour favoriser une identité européenne intolérante vis-à-vis de l'extérieur :

Magali - Moi je parle par rapport à des références historiques où l'école a été pendant un temps un vrai formateur de petits soldats. Ouais, c'est un peu dans cette idée là de dire que si l'école forme des Européens, c'est des Européens ouverts, pas des Européens... Je suis prudente contre l'utilisation qui peut être faite de l'école en fait. Bon les programmes actuels tels que je les perçois moi me semblent pas être... Mais c'est... Ouais, j’ai une espèce de vigilance quand même un peu vis-à-vis de... du sentiment d'appartenance à une catégorie et à un groupe et euh... et du côté groupe que l'on doit défendre, parce qu’on appartient à ce groupe là.

L'Europe suscite en fait des investissements contradictoires, au nom de la défense du même modèle civique. Armelle, Rose, Céline ou Magali témoignent d'une vision universaliste et individualiste de la citoyenneté: 
éduquer les enfants d'un point de vue civique, c'est avant tout pour elles leur apprendre à être ouvert aux autres et à les considérer comme des semblables, quelles que soient leurs appartenances. Cette conception partagée conduit pourtant à des appréciations divergentes de l'Europe, qui peut être soit considérée avec méfiance comme une nouvelle barrière à l'égard du reste du monde, soit être perçue avec enthousiasme comme symbolisant le dépassement des communautés plus restreintes. La nature même du projet européen est ici en jeu : il est en effet ambivalent de ce point de vue tant il mêle des logiques tendant à l'ouverture ou à la fermeture (Delanty, 2006).

Des enseignants proches d'un point de vue idéologique peuvent donc investir l'Europe de manière opposée, ce qui montre à quel point elle demeure en partie une construction politique floue. Le caractère indéfini du projet européen est aussi ce qui commande un troisième type d'attitude envers l'Europe, différent du rejet ou de l'adhésion : l'indifférence. Alors que le contexte des entretiens mettait en relief la question européenne, une partie des instituteurs n'évoquent l'Union européenne que lorsqu'ils sont directement interrogés sur ce thème. Leurs réponses sont brèves et dépourvues d'implication affective. L'UE n'est ici qu'un point du programme scolaire. Certains de ces enseignants disent même ne pas l'avoir abordé en classe, ne pas y avoir "pensé" . L'Europe est décrite par ces instituteurs « indifférents » comme " abstraite», "lointaine ", "vague " pour les enfants. Cette description apparaît de prime abord surprenante dans la mesure où, d'un strict point de vue géographique, l'Europe n'est pas, à la différence d'autres régions du monde, particulièrement " lointaine ». En outre, les enfants ont plus de connaissances sur l'Europe que semblent le supposer ces enseignants ${ }^{23}$. Par la télévision, Internet ou les voyages, l'image qu'ils en ont n'est pas non plus nécessairement "abstraite ", d'autant que ces enfants n'ont connu que l'euro, marque tangible et concrète de l'unification européenne dans leur vie quotidienne. Cette perception d'une Europe abstraite et lointaine reflète la diffusion, dans les milieux scolaires, du discours pédagogique qui met en avant, avec Jean Piaget, l'image d'un

\footnotetext{
23 Voir l'article de K. Throssell dans ce même numéro: son étude porte sur des enfants plus jeunes (entre 7 et 9 ans) que ceux des classes de notre enquête, or l'Europe est davantage abordée dans les dernières classes du primaire. Pourtant, même les enfants qu'elle interroge ont des connaissances sur l'Europe.
} 
enfant qui ne se décentre que progressivement de son environnement immédiat (famille, quartier, etc). Mais dans les entretiens de certains enseignants qui travaillent dans des écoles de quartiers populaires, cette idée semble aussi dotée d'une forte connotation sociale: si l'Europe est "lointaine ", c'est parce que les horizons des enfants des familles populaires s'arrêtent aux portes du quartier. Commentant la rareté des références à l'Europe et au monde dans les rédactions de ses élèves, Sabine, 28 ans, qui enseigne depuis deux ans dans une école de ZEP à Nice, évoque ainsi l'ignorance d'une partie des élèves de son école d'environnements plus larges que le quartier:

Sabine - l'Union européenne, le monde pour eux c'est un peu loin. Tu sais, $\mathrm{y}$ en a, dans les classes, ils savent même pas que leur quartier $\mathrm{X}$ fait partie de la ville de Nice, donc à partir de là... Ca leur parle pas.

Le contexte d'enseignement peut donc influer sur les pratiques éducatives relatives à l'Europe, en décourageant certains enseignants des écoles populaires de passer trop de temps sur un sujet qui leur parait éloigné des références qui font sens pour leurs élèves. Ceci est cependant aussi à relier au projet européen lui-même et à la manière dont il est présenté aux citoyens. Ces mêmes instituteurs n'hésitent pas en effet à faire découvrir à leurs élèves, dans d'autres domaines, des univers éloignés de leur monde quotidien supposé. Au-delà des différences d'attitudes, la difficulté qu'ont les enseignants à donner sens à l'Europe en classe autrement qu'en la présentant comme une association de pays nous semble donc liée au caractère encore flou du projet européen pour les citoyens d'aujourd'hui. Liée à l'intégration croissante du monde mais limitée à une zone géographique particulière, incarnant une forme de supranationalité tout en n'ayant pas fait disparaître les États-nations comme autorités politiques majeures et incarnées dans l'imaginaire citoyen, l'Union européenne reste une construction ambivalente. Les élites politiques françaises (Rozenberg, 2005) comme européennes n'ont pas construit de discours sur l'UE qui permettrait de lui donner une signification forte. Pour ces raisons, l'UE est susceptible d'être investie de manière contradictoire par des enseignants qui pourtant se ressemblent, ou d'être considérée comme un objet trop vague, pour les enfants mais aussi en premier lieu pour les instituteurs. 
Une représentation partagée: l'UE comme réalité irréversible, les citoyens impuissants

Outre leur difficulté à donner sens à une construction qui demeure en partie floue à leurs yeux, une autre logique commune est repérable dans les attitudes des enseignants à l'égard de l'Europe et pèse sur leurs pratiques éducatives: les instituteurs partagent l'idée que l'unification européenne est un processus irréversible. Quoi que l'on peut penser d'elle, on ne peut y échapper: l'UE est déjà là et elle sera, sans qu'on puisse y faire quelque chose, une part incontournable de l'environnement des enfants. C'est pour cette raison aussi qu'il faut l'enseigner, quel que soit le regard, indifférent, positif ou critique, que l'on porte sur elle. Cette représentation sous-jacente est repérable dans de nombreux entretiens, y compris dans ceux d'enseignants très investis dans l'enseignement de l'Union européenne: pour Monique, par exemple, "on l'a, l'Union européenne, qu'on le veuille ou non ". L'unification européenne est un processus sur lequel les enseignants sentent qu'ils ont peu de prise, pas plus qu'en auront leurs élèves plus tard. Les instituteurs n'en tirent pas un discours revendicatif: ils manifestent par leurs propos la dépossession des citoyens que représente encore aujourd'hui l'Union européenne. C'est sans doute ce sentiment diffus d'impuissance qui permet en partie de comprendre pourquoi les enseignants présentent peu l'Union européenne comme un système politique, n'en font pas ou peu étudier les institutions et ne mettent pas l'accent sur l'idée de citoyenneté européenne dans leurs pratiques d'enseignement effectives.

Le poids des programmes scolaires et des routines professionnelles: le tropisme national non contesté

La convergence relative des pratiques d'enseignement est aussi en grande partie à rapporter au contexte professionnel dans lequel sont plongés les enseignants. Les instituteurs sont tenus de suivre des programmes scolaires, qui, tout en leur laissant une marge d'action importante compte tenu de leur caractère sommaire, leur servent de cadre de référence. Ils utilisent également des manuels scolaires et d'autres outils pédagogiques pour préparer leurs séances. D'un ouvrage pédagogique à l'autre, il existe des différences certaines dans le mode de présentation de l'Europe et dans l'image de la nation qui est construite. 
Cependant, ces ouvrages suivent dans une large mesure le découpage et les sujets d'étude identifiés dans les programmes scolaires, et, à leur image, insistent dans l'ensemble peu sur l'Europe comme communauté politique. De la même manière, conformément aux textes officiels, le cadre national est dans ces ouvrages la référence dominante, à la fois comme lieu de l'histoire et espace de citoyenneté.

L'ouverture des programmes scolaires à une dimension européenne et mondiale étant récente et limitée, la socialisation professionnelle des instituteurs les a habitués à ce tropisme national. En outre, la plupart des instituteurs ont eux-mêmes connu, par leur propre socialisation scolaire, un enseignement quasi-exclusivement axé sur le national. En définitive, la prédominance du national dans l'enseignement nous paraît en grande partie relever d'effets de routine et d'un processus de reproduction impensé. Les entretiens font apparaitre de profondes divergences entre les instituteurs sur la question de l'appartenance nationale, à la fois sur son importance et sur son contenu. Mais ces clivages n'ont pas l'implication qu'on pourrait supposer en matière de pratiques d'enseignement, pratiques qui restent en grande partie dominées par des routines professionnelles. Au niveau des textes officiels, le cadrage national des contenus d'enseignement a fait l'objet d'une démarche consciente et volontaire. Du côté des instituteurs, des logiques et des routines professionnelles sont en jeu, et elles sont différentes de celles qui président à l'élaboration des politiques scolaires et à la formulation publique des problèmes éducatifs (Balland, 2009). Les instituteurs de notre enquête ne semblent pas s'interroger beaucoup sur le sens et les conséquences de leurs pratiques d'enseignement pour la socialisation civique des enfants. Ils manquent de temps pour la réflexion pédagogique et, à l'école élémentaire, c'est l'acquisition des compétences fondamentales et instrumentales, en français et en mathématiques, qui les préoccupe le plus. Il faut dire aussi, comme on l'a déjà souligné, que l'idée que l'école primaire doive avant tout apprendre à l'enfant à appartenir à la nation n'a jamais fait l'objet de contestations fortes et visibles dans le débat public et passe au contraire souvent pour une évidence ${ }^{24}$. Les enseignants du primaire

24 Le débat actuel sur l'identité nationale le montre bien. Si le sens et le contenu de "l'identité nationale » sont débattus, l'idée qu'une des finalités majeures de l'école est de contribuer à sa construction est peu remise en cause. Ce sont les pratiques d'enseignement 
ne trouvent donc pas, dans leur environnement professionnel et plus largement, sociopolitique, d'éléments forts pouvant les conduire à questionner les référents identitaires qu'ils produisent par leur enseignement. Qu'ils valorisent la nation ou une collectivité universelle, les instituteurs continuent ainsi à nourrir par leurs pratiques d'enseignement, sans avoir pleinement conscience des implications identitaires et civiques de ces pratiques, l'apprentissage de l'appartenance à la nation.

\section{L'Europe et la politique: frein à l'enseignement et dépolitisation}

Le poids des routines professionnelles ne concerne pas seulement la persistance du national dans les contenus d'enseignement. Il semble aussi se manifester dans l'évitement de la dimension politique de l'Union européenne.

Une partie des enseignants font part de leur difficulté à parler d'un tel sujet en classe parce que l'UE fait l'objet de conflits politiques, en particulier dans le contexte spécifique pendant lequel se sont déroulés les entretiens. Ils craignent que leur propre opinion se donne à voir dans la présentation qu'ils pourraient faire du sujet. Cela les conduit à éviter certaines discussions avec les élèves, ou à présenter l'Union européenne essentiellement sous un jour factuel, voire à ne pas en parler du tout en classe. Gaëlle, on l'a vu, juge le traitement du sujet " compliqué ». Elle refuse de faire part à ses élèves de son opinion négative sur les orientations actuelles de l'UE et reconnaît qu'elle aborde le thème " assez froidement» en classe. René, 54 ans, enseignant à Nice dans une école mixte socialement, dit ne pas parler de l'Europe aux élèves car il s'agit d'un "sujet brûlant». Un peu plus tôt lors de l'entretien, il employait la même expression pour l'histoire après 1945, qu'il choisit de ne pas enseigner parce que " ça peut être considéré comme politique ". Marc, enseignant dans une école de ZEP située à Nantes, qui dit ne plus voter depuis longtemps mais se sentir proche de l'extrême gauche aujourd'hui, fait part en entretien de ses réserves sur l'Union européenne actuelle. Interrogé sur l'enseignement de l'Europe à l'école, il évoque les difficultés

proposées (par exemple le chant de la Marseillaise par les élèves, proposé par Eric Besson, voir le site officiel du débat:) et leur signification pour l'image qui est donnée de la nation qui sont les plus contestées. La faiblesse des discussions sur l'Europe dans ce débat est frappante. 
pouvant entourer la discussion sur ce sujet en classe. Il juge également le sujet "compliqué" (il le présente d'ailleurs comme tel à ses élèves). Il en parle en classe, mais présente l'UE avant tout à travers ses différents pays et comme une réalité économique. Aller plus loin présenterait le risque d'introduire la politique en classe. Marc donne en exemple la question de l'entrée de la Turquie dans l'Union européenne:

Marc-C'est pas un truc [parler de l'Europe à l'école]... c'est pas un truc facile parce qu'à cet âge là les gamins ils posent plein de questions. Et quand y'en a un "la Turquie va entrer dans l'Europe ", c'est pas facile de répondre parce que y'aurait une dose de politique dans la réponse et même si on dit qu'on peut parler de politique, faut quand même éviter, même si c'est à la fois sans prendre parti et sans donner des éléments qui sont des prises de parti de certains de nos dirigeants, on a vite fait d'avoir peur de dire une bêtise ou de dire quelque chose qui puisse traduire une interprétation.

Les enseignants assument cependant parfois leur prise de position, en évoquant leur engagement pro-européen pour expliquer leur implication dans l'enseignement de l'Europe ou dans des projets européens. Mais leur mode de présentation de l'UE contribue largement à la dépolitiser. L'Europe est certes présentée ici de manière très positive, mais elle est aussi décrite comme une réalité factuelle: elle n'est jamais évoquée comme un projet dont les finalités sont controversées et qui fait l'objet de conflits dans le monde politique.

Cette dépolitisation de l'Union européenne se retrouve dans d'autres secteurs de l'action publique ${ }^{25}$. Dans le contexte scolaire, elle renvoie cependant à des logiques spécifiques, et en premier lieu aux réticences des enseignants face à l'entrée de la politique dans la classe. La norme de neutralité politique, qui relève largement dans le monde scolaire d'une routine rhétorique et pratique, signifie bien plus que la nonexpression par l'enseignant de son opinion personnelle. Dans la pratique, les discours tenus en classe et les enseignements ont un contenu politique manifeste. Mais les instituteurs évitent toutefois largement de discuter en classe des enjeux qui font l'objet de controverses politiques bien visibles dans le débat public et/ou n'en présentent aux enfants que les aspects les plus factuels ou les plus consensuels. Et ces

25 Par exemple au niveau des politiques publiques de développement local, marquées par le rôle important joué par une expertise largement dépolitisée (Guérin-Lavignotte, 1999). 
enjeux ne sont pas rapportés aux acteurs et aux groupes qui se mobilisent à leur sujet dans la sphère politique (Bozec, 2008). La présentation de l'Europe à l'école est révélatrice de ces logiques.

\section{Conclusion}

L'Union européenne a pris une place croissante dans les instructions officielles, et elle est aujourd'hui la plupart du temps un objet d'enseignement dans les classes. Aux deux niveaux, l'Union européenne apparaît essentiellement comme un groupe de pays et non comme une nouvelle identité civique pour le futur citoyen.

Un des éléments clefs permettant de rendre compte du faible sens civique et identitaire que revêt l'Union européenne est la persistance du cadre national, dans les textes officiels comme dans les classes. Les logiques à l'œuvre ne sont pas cependant les mêmes d'une sphère à l'autre. Dans les programmes scolaires, le privilège accordé au cadre national repose sur une démarche volontaire et consciente, tandis quau niveau des enseignants, il est avant tout affaire de routines professionnelles.

La convergence relative que l'on observe dans les pratiques d'enseignement traitant de l'UE semble en partie liée aux programmes scolaires et au contenu des ouvrages pédagogiques, qui mettent en avant une approche par les pays et insistent moins sur l'UE comme communauté politique. En outre, ces pratiques scolaires ne sont pas incohérentes avec la manière dont est perçue l'Europe par les enseignants: travailler sur les différents pays de l'Union européenne permet à la fois ne pas s'engager sur le terrain politique, de tenter de refléter, même de façon limitée, la diversité culturelle en son sein ou de la " rapprocher » des enfants en lui donnant un sens plus concret. C'est aussi la faible lisibilité du projet européen pour les citoyens en général - et les enseignants ne se démarquent pas en cela de leurs co-citoyens - qui le rend si difficile à traduire sur un plan pédagogique autrement que comme une collection de pays.

Seuls les enseignants les plus favorables à l'UE, en raison de leurs orientations et de leur idéologie politiques, juxtaposent à cette approche par les pays un discours positif plus spécifique sur l'Europe, qui mêle des références à la paix, à la démocratie, à la solidarité économique et à la diversité culturelle. Le discours sur l'Europe comme dépassement des communautés est également susceptible d'avoir eu une résonance dans 
les propos tenus en classe. Cependant, dans toutes ces classes aussi, l'Europe est peu présentée comme une communauté de citoyens. Quelle que soit leur position sur l'Union européenne (adhésion, méfiance ou indifférence), les enseignants partagent en fait l'idée que l'Europe est une réalité irréversible, sur laquelle les citoyens ont peu de prise.

\section{RÉFÉRENCES BIBLIOGRAPHIQUES}

Abélès Marc et Bellier Irène (1996), "La Commission européenne: du compromis culturel à la culture politique du compromis », Revue française de science politique, vol. 46, $\mathrm{n}^{\circ}$ 3, p. 431-456.

Baeyens Hélène (2000), Les Stratégies de socialisation scolaire à l'unification européenne: une dynamique saisie à partir des programmes et manuels scolaires de géographie, d'histoire et d'éducation civique des années 1950 à 1998, Thèse de Doctorat de Science Politique, Université de Grenoble 2.

Balland Ludivine, Une sociologie politique de la crise de l'Ecole: de la réussite d'un mythe aux pratiques enseignantes, Thèse de doctorat de science politique, Université de Paris X-Nanterre.

Baubérot Jean (1997), La Morale lä̈que contre l'ordre moral, Paris, Seuil.

Baugnet Lucy (2006), "Les configurations identitaires ", in Alexandre Dorna (dir.), Pour une psychologie politique française, Paris, Éditions In Press, vol. 1.

Belot Céline (2000), L'Europe en citoyenneté, Thèse de doctorat de science politique, Université Pierre Mendès France, Grenoble.

Bergounioux Alain, Cauchy Pascal, Sirinelli Jean-François et Wirth Laurent (dir.), «Faire " des Européens? L'Europe dans l'enseignement de l'histoire, de la géographie et de l'éducation civique. Actes des journées d'études des 22 janvier et 19 mars 2005, Paris, Delagrave.

Bozec Géraldine (2008) «L’enfance et le politique », La Pensée, n 354.

CEVIPOF/ministère de l'Intérieur (2007), Enquête post-électorale présidentielle 2007, vol. 1 et 2, consultable en ligne: http://www.cevipof.mshparis.fr/PEF/2007.htm

Chanet Jean-François (1996), L'École républicaine et les petites patries, Paris, Aubier.

Citron Suzanne (2008), Le Mythe national: l'histoire de France revisitée, Paris, Les Éditions de l'Atelier-Les Éditions ouvrières.

Dancel Brigitte (1999), « L'École de la Troisième République dans une France " une et indivisible »", in Claude Carpentier (coord.), Identité nationale et enseignement de l'histoire. Contextes européens et africains, Paris, L'Harmattan, p. 51-65. 
Delanty Gerard (2006), "Borders in a Changing Europe: Dynamics of Openness and Closure ", Comparative European Politics, vol. 4, p. 183-202.

Déloye Yves (1994), École et citoyenneté. Lindividualisme républicain de Jules Ferry à Vichy, Paris, Presses de la FNSP.

Déloye Yves (2007), Sociologie historique du politique, Paris, La Découverte.

Easton David (1965), A Systems Analysis of Political Life, New York, John Wiley and Sons.

Ferro Marc (1981), Comment on raconte l'histoire aux enfants à travers le monde entier, Paris, Payot.

Fumat Yveline (1978), « La socialisation politique à l'école du « Tour de France de deux enfants " aux manuels de 1977 ", Revue française de pédagogie, juillet-septembre, p. 71-82.

Green Andy (1992), Education and State Formation. The rise of Education Systems in England, France and the USA, Londres, Marc Millan Press.

Gellner Ernest (1989), Nations et nationalismes, Paris, Payot.

Girault René (1983), L'Histoire et la géographie en question: rapport au ministre de l'Education Nationale, Paris, CNDP.

Girod Marion (2006), Les Politiques d'éducation à la citoyenneté européenne: étude comparée France-Angleterre, Thèse de doctorat de science politique, Université Robert Schuman, Strasbourg.

Guérin-Lavignotte Élodie (1999), Expertise et politiques européennes de développement local, Paris, L'Harmattan.

Lelièvre Claude (2001), "Culture et nation dans l'école de la République une et indivisible ", in Claude Charpentier (coord.), Contenus d'enseignement dans un monde en mutation: permanences et ruptures, Paris, L'Harmattan, p. 331-341.

Ministère de l'Éducation nationale (1995), Programmes de l'école primaire, Paris, CNDP.

Ministère de l'Éducation nationale (2002a), Qu'apprend-on à l'école élémentaire? Les nouveaux programmes, Paris, CNDP/XO Éditions.

Ministère de la Jeunesse, de l'Éducation nationale et de la Recherche/Direction de l'enseignement scolaire (2002b), Histoire et géographie. Cycle des approfondissements (cycle 3), Paris, CNDP.

Nora Pierre (1986), «L'histoire de France de Lavisse », in Pierre Nora (dir.), Les Lieux de mémoire, tome 2, "La Nation », Paris, Gallimard.

Ozouf Jacques et Ozouf Mona (1964), "Le thème du patriotisme dans les manuels scolaires », Le Mouvement social, octobre-décembre, p. 5-31.

Percheron Annick (1978), Les 10-16 ans et la politique, Paris, PFNSP.

Perrot Danièle (1973), La Thématique politique des manuels d'histoire du cours élémentaire de l'enseignement public, Mémoire pour le DES de science politique, Université de Rennes 1. 
Pingué Daniel et Joutard Philippe (2004), "L'histoire dans les nouveaux programmes (2002) de l'école primaire. Entretien avec Philippe Joutard ", Cahiers d'histoire, ${ }^{\circ} 93$.

Rozenberg Olivier (2005), Le Parlement français et l'Union européenne (19932005): l'Europe saisie par les rôles parlementaires, Thèse de doctorat de science politique, Institut d'études politiques de Paris.

Schissler Hanna et Soysal Yasemin Nuhoglu (2005), The Nation, Europe and the World: Textbooks and Curricula in Transition, New York- Oxford, Berghahn Books.

Thiesse Anne-Marie (1997), Ils apprenaient la France: l'exaltation des régions dans le discours patriotique, Paris, Éditions de la MSH.

Thiesse Anne-Marie (2001), La Création des identités nationales: XVIII ${ }^{e}-X X^{e}$ siècle, Paris, Seuil.

Throssell Katharine (2007) "European Childhood, or a Study of how "they" are "us" ", communication au deuxième colloque international de la Section d'études européennes de l'AFSP, Amours et désamours entre Européens. Pour une sociologie politique des sentiments dans l'intégration européenne, 67 décembre, Grenoble. 